\title{
Bundle Convergence in a von Neumann Algebra and in a von Neumann Subalgebra
}

\author{
by \\ Barthélemy LE GAC and Ferenc MÓRICZ \\ Presented by Stanistaw WORONOWICZ
}

\begin{abstract}
Summary. Let $H$ be a separable complex Hilbert space, $\mathcal{A}$ a von Neumann algebra in $\mathcal{L}(H), \phi$ a faithful, normal state on $\mathcal{A}$, and $\mathcal{B}$ a commutative von Neumann subalgebra of $\mathcal{A}$. Given a sequence $\left(X_{n}: n \geq 1\right)$ of operators in $\mathcal{B}$, we examine the relations between bundle convergence in $\mathcal{B}$ and bundle convergence in $\mathcal{A}$.
\end{abstract}

1. Introduction. Bundle convergence in von Neumann algebras was introduced in 1996 by Hensz, Jajte and Paszkiewicz in their fundamental paper [2]. We refer to [2] for the definitions and basic properties of bundle convergence.

Let $H$ be a separable complex Hilbert space, $\mathcal{L}(H)$ the algebra of all bounded linear operators acting on $H, \mathcal{A}$ a von Neumann algebra in $\mathcal{L}(H)$, $\phi$ a faithful, normal state on $\mathcal{A}$, and $\mathcal{B}$ a von Neumann subalgebra of $\mathcal{A}$. Clearly, the restriction of $\phi$ to $\mathcal{B}$ defines a faithful, normal state on $\mathcal{B}$. Thus, the following question seems to be quite natural.

Question. Let $\left(X_{n}: n \geq 1\right)$ be a sequence of operators in $\mathcal{B}$ which is bundle convergent to $O$ in $\mathcal{B}$, where $O$ is the zero operator acting on $H$. Is then $\left(X_{n}\right)$ bundle convergent in $\mathcal{A}$ ?

2000 Mathematics Subject Classification: Primary 46L53, 46L10.

Key words and phrases: von Neumann algebra and subalgebra, faithful and normal state, bundle convergence, almost uniform convergence, conditional expectation in a von Neumann algebra.

This research was completed while the second named author was a visiting professor at the University of Tennessee, Knoxville during the fall semester in 2003; and it was also partially supported by the Hungarian National Foundation for Scientific Research under Grants TS 044782 and T 046192. 
We shall see in Section 2 that the answer to this question is negative in general. However, the answer is yes in the following two particular cases:

(i) If $\mathcal{A}:=L_{\infty}(\Omega, \mathcal{F}, \mu)$, where $(\Omega, \mathcal{F}, \mu)$ is a classical probability space, and $\phi$ is defined by

$$
\phi(A):=\int_{\Omega} A(\omega) d \mu(\omega), \quad A \in \mathcal{A},
$$

then the notion of bundle convergence in $\mathcal{A}$ coincides with that of almost sure convergence with respect to the probability measure $\mu$. The positive answer to the above question follows from the well known fact that in this case, any von Neumann subalgebra is of the form $L_{\infty}(\Omega, \mathcal{G}, \mu)$, where $\mathcal{G}$ is a $\sigma$-subalgebra of $\mathcal{F}$.

(ii) If the sequence $\left(X_{n}: n \geq 1\right)$ is bounded in operator norm; this follows from the fact that bundle convergence in $\mathcal{A}$ (respectively, in $\mathcal{B}$ ) is equivalent to almost uniform convergence in $\mathcal{A}$ (respectively, in $\mathcal{B}$ ), by $[2$, Properties 3.7 and Theorem 4.1].

In this paper, we deal only with a commutative von Neumann subalgebra $\mathcal{B}$ of $\mathcal{A}$. In Section 2, we study a particular case of $\mathcal{A}$ which will be useful to construct counterexamples. In Section 3, we state some relations concerning bundle convergence of subsequences, and we consider the converse problem. Namely, assuming that a sequence $\left(X_{n}\right)$ of operators in $\mathcal{B}$ is bundle convergent in $\mathcal{A}$, is it also bundle convergent in $\mathcal{B}$ ? It turns out that the answer depends on whether there exists a conditional expectation with respect to $\phi$ from $\mathcal{A}$ to $\mathcal{B}$. On closing, we raise two problems.

2. A particular case. Let $H$ be a separable complex Hilbert space and fix an orthonormal basis $\left(e_{j}: j \geq 1\right)$ in $H$. We define a faithful, normal state $\phi$ on $\mathcal{A}:=\mathcal{L}(H)$ in the following way:

$$
\phi(A):=\sum_{j=1}^{\infty} 2^{-j}\left(A e_{j} \mid e_{j}\right), \quad A \in A,
$$

where $(\cdot \mid \cdot)$ is the inner product in $H$. In fact, $\phi$ is clearly a positive, linear functional on $\mathcal{L}(H)$, for the identity operator $I$ we have $\phi(I)=1$, and $\phi$ is faithful (since $2^{-j}>0$ for all $j$ ). The normality of $\phi$ is a consequence of [3, Theorem, p. 121]. Let $\mathcal{D}$ be the von Neumann subalgebra of $\mathcal{L}(H)$ consisting of the operators in $\mathcal{L}(H)$ whose matrices are diagonal with respect to the orthonormal basis $\left(e_{j}: j \geq 1\right)$. Thus, every $X \in \mathcal{D}$ is of the form

$$
X=\sum_{j=1}^{\infty} a_{j} P_{e_{j}}, \quad \text { where }\left(a_{j}\right) \in \ell_{\infty}
$$

and $P_{e_{j}}$ is the (orthogonal) projection on the line $\mathbb{C} e_{j}$. 
Now, for every $\alpha:=\left(\alpha_{1}, \alpha_{2}, \ldots\right) \in \ell_{2}, \alpha \neq(0,0, \ldots)$, let us define a vector $u$ depending on $\alpha$ as follows:

$$
u:=K \sum_{j=1}^{\infty} \alpha_{j} 2^{-j / 2} e_{j},
$$

where the constant $K>0$ is chosen so that $\|u\|=1$. Denote by $P_{u}$ the projection on the line $\mathbb{C} u$.

Theorem 1. The projection $P_{u}$ belongs to each bundle in $\mathcal{L}(H)$.

Proof. Let $\mathcal{P}$ be a bundle in $\mathcal{L}(H)$. By definition, $\mathcal{P}$ is determined by some sequence $\left(D_{n}: n \geq 1\right)$ of positive operators in $\mathcal{L}(H)$ such that

$$
\sum_{n=1}^{\infty} \phi\left(D_{n}\right)<\infty
$$

We associate with each operator $D_{n}$ its infinite matrix $\left(d_{n, j, k}\right)$ in the orthonormal basis $\left(e_{j}\right)$, where

$$
d_{n, j, k}:=\left(D_{n} e_{k} \mid e_{j}\right), \quad n, j, k=1,2, \ldots
$$

Taking into account that by the positivity of $D_{n}$,

$$
D_{n}=C_{n}^{*} C_{n} \quad \text { for some } C_{n} \in \mathcal{L}(H),
$$

where $C_{n}^{*}$ is the adjoint operator to $C_{n}$, and making use of the CauchySchwarz inequality, we conclude that

$$
\left|d_{n, j, k}\right|^{2} \leq d_{n, j, j} d_{n, k, k}, \quad n, j, k=1,2, \ldots
$$

By (2.1) and (2.4), we may write

$$
\phi\left(D_{n}\right)=\sum_{j=1}^{\infty} 2^{-j} d_{n, j, j}, \quad n=1,2, \ldots .
$$

Let $x$ be an arbitrary vector in $H$. Then

$$
x=\sum_{j=1}^{\infty} x_{j} e_{j} \quad \text { for some }\left(x_{j}\right) \subset \ell_{2} .
$$

Since $\|u\|=1$, we have $P_{u} x=(x \mid u) u$ and thus

$$
\begin{aligned}
D_{n} P_{u} x & =(x \mid u) D_{n} u=K(x \mid u) \sum_{j=1}^{\infty} \alpha_{j} 2^{-j / 2} D_{n} e_{j} \\
& =K(x \mid u) \sum_{j=1}^{\infty} \alpha_{j} 2^{-j / 2} \sum_{k=1}^{\infty}\left(D_{n} e_{j} \mid e_{k}\right) e_{k} \\
& =K(x \mid u) \sum_{k=1}^{\infty}\left(\sum_{j=1}^{\infty} \alpha_{j} 2^{-j / 2} d_{n, k, j}\right) e_{k} .
\end{aligned}
$$


Accordingly, we define

$$
y_{n}:=\sum_{k=1}^{\infty} y_{n, k} e_{k}, \quad y_{n, k}:=\sum_{j=1}^{\infty} \alpha_{j} 2^{-j / 2} d_{n, k, j}, \quad n, k=1,2, \ldots
$$

Thus, we can rewrite $(2.7)$ in the form

$$
D_{n} P_{u} x=K(x \mid u) y_{n}
$$

whence

$$
P_{u} D_{n} P_{u} x=K(x \mid u) P_{u} y_{n}=K(x \mid u)\left(y_{n} \mid u\right) u ;
$$

in particular,

$$
\left\|P_{u} D_{n} P_{u} x\right\|=K|(x \mid u)| \cdot\left|\left(y_{n} \mid u\right)\right|, \quad n=1,2, \ldots
$$

Now, we estimate $\left|\left(y_{n} \mid u\right)\right|$. By (2.2) and (2.8), we have

$$
\begin{aligned}
\left(y_{n} \mid u\right) & =\sum_{k=1}^{\infty} y_{n, k}\left(e_{k} \mid u\right)=K \sum_{k=1}^{\infty} y_{n, k} \alpha_{k} 2^{-k / 2} \\
& =K \sum_{k=1}^{\infty}\left(\sum_{j=1}^{\infty} \alpha_{j} 2^{-j / 2} d_{n, k, j}\right) \alpha_{k} 2^{-k / 2}
\end{aligned}
$$

By (2.5), we find that

$$
\begin{aligned}
\left|\left(y_{n} \mid u\right)\right| & \leq K \sum_{k=1}^{\infty}\left(\sum_{j=1}^{\infty}\left|\alpha_{j}\right| 2^{-j / 2}\left|d_{n, k, j}\right|\right)\left|\alpha_{k}\right| 2^{-k / 2} \\
& \leq K \sum_{k=1}^{\infty}\left(\sum_{j=1}^{\infty}\left|\alpha_{j}\right| 2^{-j / 2} \sqrt{d_{n, j, j}}\right)\left|\alpha_{k}\right| 2^{-k / 2} \sqrt{d_{n, k, k}} \\
& =K\left(\sum_{k=1}^{\infty}\left|\alpha_{k}\right| 2^{-k / 2} \sqrt{d_{n, k, k}}\right)^{2}
\end{aligned}
$$

Applying the Cauchy inequality, by (2.6) and (2.10), we conclude that

$$
\left|\left(y_{n} \mid u\right)\right| \leq K\|\alpha\|_{2}^{2} \phi\left(D_{n}\right), \quad n=1,2, \ldots,
$$

where $\|\alpha\|_{2}$ is the $\ell_{2}$-norm of $\alpha=\left(\alpha_{1}, \alpha_{2}, \ldots\right)$. Combining (2.9) and (2.11) gives

$$
\left\|P_{u} D_{n} P_{u} x\right\| \leq K^{2}\|\alpha\|_{2}^{2}\|x\| \phi\left(D_{n}\right) .
$$

Since $x \in H$ is arbitrary, we have

$$
\left\|P_{u} D_{n} P_{u}\right\|_{\infty} \leq K^{2}\|\alpha\|_{2}^{2} \phi\left(D_{n}\right), \quad n=1,2, \ldots
$$

By (2.3), it follows that $\left\|P_{u} D_{n} P_{u}\right\|_{\infty} \rightarrow 0$ as $n \rightarrow \infty$. An analogous argument shows that

$$
\sup _{n \geq 1} \sum_{k=1}^{n}\left\|P_{u} D_{k} P_{u}\right\|_{\infty}<\infty \text {. }
$$


Consequently, the projection $P_{u}$ belongs to the bundle determined by $\left(D_{n}\right)$, as claimed.

Now, let $\left(X_{n}: n \geq 1\right)$ be a sequence of operators in $\mathcal{D}$. We shall examine the relations between bundle convergence in $\mathcal{D}$ and in $\mathcal{A}=\mathcal{L}(H)$. First, we need the following

Lemma. Let $\left(X_{n}\right)$ be a sequence in $\mathcal{D}$. Then

$$
X_{n} \stackrel{\mathrm{b}, \mathcal{D}}{\longrightarrow} O \quad \text { as } n \rightarrow \infty
$$

if and only if

$$
\left(X_{n} e_{j} \mid e_{j}\right) \rightarrow 0 \text { as } n \rightarrow \infty \text { for each } j=1,2, \ldots \text {. }
$$

Proof. We may identify $\mathcal{D}$ with the $L_{\infty}$-space of the probability space $(\mathbb{N}, \mathcal{F}, \mu)$, where $\mathbb{N}$ is the set of natural numbers, $\mathcal{F}$ is the family of all subsets of $\mathbb{N}$, and $\mu$ is given by

$$
\mu(\{j\})=2^{-j}, \quad j=1,2, \ldots
$$

Thus, bundle convergence in $\mathcal{D}$ coincides with almost sure convergence with respect to $\mu$ (see, for example, [2, p. 29]).

Corollary 1. Let $\left(X_{n}\right)$ be a sequence in $\mathcal{D}$. Then

$$
X_{n} \stackrel{\mathrm{b}, \mathcal{A}}{\longrightarrow} O \quad \text { implies } \quad X_{n} \stackrel{\mathrm{b}, \mathcal{D}}{\longrightarrow} O \quad \text { as } n \rightarrow \infty .
$$

Proof. Fix $j=j_{0} \geq 1$. In (2.2), we choose $\left(\alpha_{1}, \alpha_{2}, \ldots\right)$ as follows:

$$
\alpha_{j_{0}}=2^{j_{0} / 2}, \quad \alpha_{j}=0 \quad \text { if } j \neq j_{0} .
$$

Thus $u=e_{j_{0}}$. We deduce that

$$
X_{n} P_{u} u=X_{n} e_{j_{0}}=\left(X_{n} e_{j_{0}} \mid e_{j_{0}}\right) e_{j_{0}} .
$$

Hence we get

$$
\left|\left(X_{n} e_{j_{0}} \mid e_{j_{0}}\right)\right| \leq\left\|X_{n} P_{e_{j_{0}}}\right\|_{\infty} \rightarrow 0 \quad \text { as } n \rightarrow \infty,
$$

by Theorem 1 . Then $X_{n} \stackrel{\mathrm{b}, \mathcal{D}}{\longrightarrow} 0$, as a consequence of the lemma.

Corollary 2. There exists a sequence $\left(X_{n}\right)$ in $\mathcal{D}$ which is bundle convergent to $O$ in $\mathcal{D}$, but fails to be bundle convergent in $\mathcal{L}(H)$.

Proof. Let

$$
X_{n}:=n 2^{n / 2} P_{e_{n}}, \quad n=1,2, \ldots
$$

Then $X_{n} \in \mathcal{D}$ and $X_{n} \stackrel{\text { b, } \mathcal{D}}{\longrightarrow} O$ as $n \rightarrow \infty$, since for every $j=1,2, \ldots$, we have $\left(X_{n} e_{j} \mid e_{j}\right)=0$ as soon as $n>j$. Now, in (2.2) choose

$$
u:=K \sum_{j=1}^{\infty} j^{-1} 2^{-j / 2} e_{j} ;
$$


it follows that

$$
X_{n} P_{u} u=X_{n} u=K e_{n}, \quad n=1,2, \ldots
$$

By using (2.13) and the orthonomality of the system $\left(e_{j}\right)$, we get

$$
\begin{aligned}
\left\|\left(X_{n+1}-X_{n}\right) P_{u}\right\|_{\infty} & \geq\left\|\left(X_{n+1}-X_{n}\right) P_{u} u\right\| \\
& =K\left\|e_{n+1}-e_{n}\right\|=K \sqrt{2}, \quad n=1,2, \ldots
\end{aligned}
$$

Consequently, if $\left(X_{n}\right)$ were bundle convergent in $\mathcal{L}(H)$ to some operator $X$, then $\left(X_{n+1}-X_{n}: n \geq 1\right)$ would be bundle convergent to $O$ in $\mathcal{L}(H)$, due to the additivity of bundle convergence; in particular, we would have

$$
\left\|\left(X_{n+1}-X_{n}\right) P_{u}\right\|_{\infty} \rightarrow 0 \text { as } n \rightarrow \infty,
$$

since $P_{u}$ belongs to every bundle in $\mathcal{L}(H)$. But this contradicts (2.14), and the contradiction yields the conclusion of Corollary 2.

REMARK 1. The sequence

$$
X_{n}:=n 2^{n / 2} P_{e_{n}}, \quad n=1,2, \ldots,
$$

converges almost uniformly to $O$ in $\mathcal{D}$; consequently, it converges almost uniformly to $O$ in $\mathcal{L}(H)$, as well. In this way, we have obtained a simple example which illustrates the following known statement.

Corollary 3. There exists a sequence $\left(X_{n}: n \geq 1\right)$ of operators in $\mathcal{L}(H)$ such that $\left(X_{n}\right)$ converges almost uniformly, but fails to be bundle convergent in $\mathcal{L}(H)$.

A more theoretic proof of Corollary 3 can be derived from [6, Proposition 4.6], where it is proved that almost uniform convergence (unlike bundle convergence) does not have the additivity property.

COROLlary 4. There exists a sequence $\left(Y_{n}: n \geq 1\right)$ of operators in $\mathcal{L}(H)$ such that $\left(Y_{n}\right)$ is bundle convergent to $O$, but $\left(Y_{n}^{2}\right)$ fails to be bundle convergent in $\mathcal{L}(H)$.

Proof. Let $\left(X_{n}\right)$ be given by (2.15) and

$$
Y_{n}:=X_{n}^{1 / 2}=n^{1 / 2} 2^{n / 4} P_{e_{n}}, \quad n=1,2, \ldots
$$

By (2.1), we have

$$
\phi\left(Y_{n}^{2}\right)=\phi\left(X_{n}\right)=n 2^{n / 2} \phi\left(P_{e_{n}}\right)=n 2^{-n / 2} .
$$

Since

$$
\sum_{n=1}^{\infty} \phi\left(Y_{n}^{2}\right)=\sum_{n=1}^{\infty} n 2^{-n / 2}<\infty,
$$

by [2, Proposition 3.1] we conclude that $\left(Y_{n}\right)$ is bundle convergent to $O$ as $n \rightarrow \infty$. But we have seen in the proof of Corollary 2 that the sequence $\left(Y_{n}^{2}=X_{n}: n \geq 1\right)$ fails to be bundle convergent in $\mathcal{L}(H)$. 
3. Bundle convergence of subsequences. The sequence $\left(X_{n}: n \geq 1\right)$ we used in the proof of Corollary 2 does not admit a subsequence $\left(X_{n_{k}}\right.$ : $k \geq 1$ ) bundle convergent in $\mathcal{L}(H)$, since, with $u$ given by $(2.12)$,

$$
\left\|\left(X_{n_{k+1}}-X_{n_{k}}\right) P_{u}\right\|_{\infty} \geq K\left\|e_{n_{k+1}}-e_{n_{k}}\right\|=K \sqrt{2}, \quad k=1,2, \ldots
$$

So the following result is of some interest.

Theorem 2. Let $H$ be a separable complex Hilbert space, $\mathcal{A}$ a von Neumann algebra in $\mathcal{L}(H)$, $\phi$ a faithful, normal state on $\mathcal{A}$, and $\mathcal{B}$ a commutative von Neumann subalgebra of $\mathcal{A}$. Let $\left(X_{n}: n \geq 1\right)$ be a sequence in $\mathcal{B}$ such that

$$
\begin{gathered}
\sup _{n \geq 1} \phi\left(\left|X_{n}\right|^{\alpha}\right)<\infty \quad \text { for some } \alpha>2, \\
X_{n} \stackrel{\mathrm{b}, \mathcal{B}}{\longrightarrow} O \quad \text { as } n \rightarrow \infty .
\end{gathered}
$$

Then there exists a subsequence $\left(X_{n_{k}}: k \geq 1\right)$ of $\left(X_{n}\right)$ such that

$$
X_{n_{k}} \stackrel{\mathrm{b}, \mathcal{A}}{\longrightarrow} O \quad \text { as } k \rightarrow \infty .
$$

Proof. There exists a probability space $(\Omega, \mathcal{F}, \mu)$ and an isomorphism $X \mapsto T_{X}$ of $\mathcal{B}$ onto $L_{\infty}(\Omega, \mathcal{F}, \mu)$ such that

$$
\phi(X)=\int_{\Omega} T_{X}(\omega) d \mu(\omega)
$$

for every $X$ in $\mathcal{B}$. Let $f_{n}:=T_{X_{n}}$. If $A$ is a measurable set in $\Omega$, then by using Hölder's inequality with $1 / p+1 / q=1, p:=\alpha / 2$, we find

$$
\begin{aligned}
\phi\left(\left|X_{n}\right|^{2}\right) & =\int_{\Omega}\left|f_{n}\right|^{2} d \mu=\int_{A}\left|f_{n}\right|^{2} d \mu+\int_{A^{\mathrm{c}}}\left|f_{n}\right|^{2} d \mu \\
& \leq \sup _{\omega \in A}\left|f_{n}(\omega)\right|^{2}+\left(\int_{\Omega}\left|f_{n}\right|^{\alpha} d \mu\right)^{2 / \alpha} \cdot \mu\left(A^{\mathrm{c}}\right)^{(\alpha-2) / \alpha} .
\end{aligned}
$$

Now, since bundle convergence in $L_{\infty}(\Omega, \mathcal{F}, \mu)$ is in fact almost sure convergence with respect to $\mu$, by using Egorov's theorem we may construct a measurable set $A$ in $\Omega$ such that $\mu\left(A^{\mathrm{c}}\right)$ is arbitrarily small and $f_{n} \rightarrow 0$ as $n \rightarrow \infty$ uniformly on $A$. Then, by using (3.1) and (3.4), we derive that

$$
\phi\left(\left|X_{n}\right|^{2}\right) \rightarrow 0 \quad \text { as } n \rightarrow \infty \text {. }
$$

By a classical argument, there exists a subsequence $\left(X_{n_{k}}: k \geq 1\right)$ of $\left(X_{n}\right)$ for which

$$
\sum_{k=1}^{\infty} \phi\left(\left|X_{n_{k}}\right|^{2}\right)<\infty .
$$

Then, by [2, Property 3.1, p. 30], we get

$$
X_{n_{k}} \stackrel{\mathrm{b}, \mathcal{A}}{\longrightarrow} O \quad \text { as } k \rightarrow \infty .
$$


Remark 2. For each $\alpha, 1 \leq \alpha<2$, we can exhibit a sequence $\left(X_{n}: n \geq 1\right)$ in the von Neumann subalgebra $\mathcal{D}$ defined in Section 2 such that

$$
\sup _{n \geq 1} \phi\left(\left|X_{n}\right|^{\alpha}\right)<\infty, \quad X_{n} \stackrel{\text { b,D }}{\longrightarrow} O \quad \text { as } n \rightarrow \infty,
$$

but $\left(X_{n}\right)$ does not admit a subsequence satisfying (3.3). To this end, let

$$
X_{n}:=2^{n / \alpha} P_{e_{n}}, \quad n=1,2, \ldots
$$

Then

$$
\phi\left(\left|X_{n}\right|^{\alpha}\right)=2^{n} \phi\left(P_{e_{n}}\right)=1 \quad \text { and } \quad X_{n} \stackrel{\mathrm{b}, \mathcal{D}}{\longrightarrow} O \quad \text { as } n \rightarrow \infty
$$

by the same argument as in the proof of Corollary 2. On the other hand,

$$
X_{n} P_{u} u=2^{n / \alpha} n^{-1} 2^{-n / 2} e_{n},
$$

where $u$ is given by (2.12). Hence

$$
\left\|X_{n} P_{u}\right\|_{\infty} \geq \frac{1}{n} 2^{n(1 / \alpha-1 / 2)} \rightarrow \infty \quad \text { as } n \rightarrow \infty .
$$

REMARK 3. The case $\alpha=2$ is open.

Theorem 3. Let $H$ be a separable complex Hilbert space, $\mathcal{A}$ a von Neumann algebra in $\mathcal{L}(H)$, $\phi$ a faithful, normal state on $\mathcal{A}$, and $\mathcal{B}$ a commutative von Neumann subalgebra of $\mathcal{A}$. Let $\left(X_{n}: n \geq 1\right)$ be a sequence in $\mathcal{B}$ such that

$$
\begin{gathered}
\sup _{n \geq 1} \phi\left(\left|X_{n}\right|\right)<\infty, \\
X_{n} \stackrel{\mathrm{b}, \mathcal{A}}{\longrightarrow} O \quad \text { as } n \rightarrow \infty .
\end{gathered}
$$

Then there exists a subsequence $\left(X_{n_{k}}\right)$ of $\left(X_{n}\right)$ such that

$$
X_{n_{k}} \stackrel{\mathrm{b}, \mathcal{B}}{\longrightarrow} O \quad \text { as } k \rightarrow \infty .
$$

Proof. By (3.6), there exists a bundle $\mathcal{P}$ in $\mathcal{A}$ such that, for each $P \in \mathcal{P}$,

$$
\left\|X_{n} P\right\|_{\infty} \rightarrow 0 \quad \text { as } n \rightarrow \infty .
$$

Let

$$
A_{n}:=\left|X_{n}\right|^{1 / 2}, \quad n=1,2, \ldots .
$$

We get for each $P \in \mathcal{P}$,

$$
\begin{aligned}
\left\|A_{n} P\right\|_{\infty}^{2} & =\left\|P A_{n}^{*} A_{n} P\right\|_{\infty}=\left\|P\left|X_{n}\right| P\right\|_{\infty} \leq\|P\|_{\infty}\left\|X_{n} P\right\|_{\infty} \\
& \leq\left\|X_{n} P\right\|_{\infty} \rightarrow 0 \quad \text { as } n \rightarrow \infty .
\end{aligned}
$$

Thus,

$$
A_{n} \stackrel{\mathrm{b}, \mathcal{A}}{\longrightarrow} O \quad \text { as } n \rightarrow \infty .
$$

By (3.5), we also have

$$
\sup _{n \geq 1} \phi\left(A_{n}^{2}\right)<\infty .
$$


Now, by using [5, Proposition, p. 451], we derive that

$$
\phi\left(A_{n}\right) \rightarrow 0 \quad \text { as } n \rightarrow \infty .
$$

Let $B_{n}:=A_{n}^{1 / 2}=\left|X_{n}\right|^{1 / 4}$; since $\phi\left(B_{n}^{2}\right) \rightarrow 0$ as $n \rightarrow \infty$, there exists a subsequence $\left(B_{n_{k}}: k \geq 1\right)$ of $\left(B_{n}\right)$ such that

$$
\sum_{k=1}^{\infty} \phi\left(B_{n_{k}}^{2}\right)<\infty
$$

It follows that

$$
B_{n_{k}}=\left|X_{n_{k}}\right|^{1 / 4} \stackrel{\mathrm{b}, \mathcal{B}}{\longrightarrow} O \quad \text { as } k \rightarrow \infty
$$

Since $\mathcal{B}$ is commutative, we may derive that $X_{n_{k}} \stackrel{\text { b, } \mathcal{B}}{\longrightarrow} O$ as $k \rightarrow \infty$. Here we took into account that $\mathcal{B}$ is isomorphic to some $L_{\infty}(\Omega, \mathcal{F}, \mu)$.

Now, the following question arises naturally: In the conclusion (3.7) of Theorem 3 , is it possible to replace the subsequence $\left(X_{n_{k}}\right)$ by the whole sequence $\left(X_{n}\right)$ ? We shall see in Theorem 4 below that the answer is positive if there exists a conditional expectation $\mathcal{E}$ with respect to $\phi$ from $\mathcal{A}$ to $\mathcal{B}$.

Before stating Theorem 4 , we note the interesting fact that it may happen that $\left(X_{n}: n \geq 1\right)$ is a sequence in $\mathcal{A}$ which is bundle convergent to $O$ in $\mathcal{A}$, but $\left(\mathcal{E}\left(X_{n}\right): n \geq 1\right)$ fails to be bundle convergent to $O$ in both $\mathcal{B}$ and $\mathcal{A}$. To see this, let $\mathcal{A}:=L_{\infty}([0,1], \mathcal{F}, \lambda)$, where $\mathcal{F}$ is the Borel field on $[0,1], \lambda$ the Lebesgue measure, $\mathcal{B}=\mathbb{C} I_{[0,1]}$, and

$$
\phi(X):=\int_{0}^{1} X(t) d t, \quad X \in \mathcal{A} .
$$

Now, the conditional expectation from $\mathcal{A}$ onto $\mathcal{B}$ is given by

$$
\mathcal{E}(X)=\phi(X) I_{[0,1]}, \quad X \in \mathcal{A} .
$$

Since bundle convergence in $\mathcal{A}$ is in fact a.e. convergence with respect to Lebesgue measure, it is easy to exhibit a sequence $\left(X_{n}: n \geq 1\right)$ such that $X_{n} \rightarrow O$ a.e. as $n \rightarrow \infty$, but $\int_{0}^{1} X_{n}(t) d t$ fails to converge in $\mathbb{C}$. (Compare $[4$, Problem 3, p. 101].)

TheOREM 4. Let $H$ be a separable complex Hilbert space, $\mathcal{A}$ a von Neumann algebra in $\mathcal{L}(H), \phi$ a faithful and normal state on $\mathcal{A}$, and $\mathcal{B}$ a commutative von Neumann subalgebra of $\mathcal{A}$ such that there exists a conditional expectation $\mathcal{E}$ with respect to $\phi$ from $\mathcal{A}$ onto $\mathcal{B}$. Then for every sequence $\left(X_{n}: n \geq 1\right)$ of operators in $\mathcal{B}$,

$$
X_{n} \stackrel{\mathrm{b}, \mathcal{A}}{\longrightarrow} O \quad \text { implies } \quad X_{n} \stackrel{\mathrm{b}, \mathcal{B}}{\longrightarrow} O \quad \text { as } n \rightarrow \infty .
$$

Proof. In fact, instead of bundle convergence, it is sufficient to assume only that the sequence $\left(X_{n}\right)$ is almost uniformly convergent to $O$ in $\mathcal{A}$. Then 
for every natural number $k$, there exists a projection $P_{k}$ in $\mathcal{A}$ such that

$$
\phi\left(P_{k}\right)>(k-1) / k \text { and }\left\|X_{n} P_{k}\right\|_{\infty} \rightarrow 0 \quad \text { as } n \rightarrow \infty .
$$

By using the properties of the conditional expectation $\mathcal{E}$ (see [7, p. 211]), we have

$$
\begin{gathered}
\left\|X_{n} \mathcal{E}\left(P_{k}\right)\right\|_{\infty}=\left\|\mathcal{E}\left(X_{n} P_{k}\right)\right\|_{\infty} \leq\left\|X_{n} P_{k}\right\|_{\infty}, \\
\mathcal{E}\left(P_{k}\right) \text { is positive, } \quad \phi\left(\mathcal{E}\left(P_{k}\right)\right)=\phi\left(P_{k}\right), \\
\left\|\mathcal{E}\left(P_{k}\right)\right\|_{\infty} \leq\left\|P_{k}\right\|_{\infty}=1
\end{gathered}
$$

We recall (cf. [1, Théorème 1, p. 118] and the proof of our Theorem 2 above) that there exist a probability space $(\Omega, \mathcal{F}, \mu)$ and an isomorphism $X \mapsto T_{X}$ of $\mathcal{B}$ onto $L_{\infty}(\Omega, \mathcal{F}, \mu)$ such that

$$
\phi(X)=\int_{\Omega} T_{X}(\omega) d \mu(\omega), \quad X \in \mathcal{B} .
$$

Then

$$
\varrho_{k}:=T_{\mathcal{E}\left(P_{k}\right)}, \quad k=1,2, \ldots,
$$

is a nonnegative function on $L_{\infty}(\Omega, \mathcal{F}, \mu)$, and it follows from (3.12) and (3.13) that

$$
\int_{\Omega} \varrho_{k}(\omega) d \mu(\omega)>(k-1) / k, \quad\left\|\varrho_{k}\right\|_{\infty} \leq 1
$$

Now, let

$$
\Omega_{k}:=\left\{\omega \in \Omega: \varrho_{k}(\omega)=0\right\}, \quad k=1,2, \ldots
$$

By (3.14), we have $\mu\left(\Omega_{k}\right) \leq 1 / k$. It follows from (3.11) that

$$
\left\|T_{X_{n}} \varrho_{k}\right\|_{\infty} \rightarrow 0 \quad \text { as } n \rightarrow \infty, k=1,2, \ldots
$$

This means that

$$
T_{X_{n}} \rightarrow O \quad \text { as } n \rightarrow \infty \text { a.e. on } \Omega_{k}^{\mathrm{c}}, k=1,2, \ldots
$$

Consequently, we have

$$
T_{X_{n}} \rightarrow O \quad \text { a.e. on } \bigcup_{k=1}^{\infty} \Omega_{k}^{\mathrm{c}},
$$

whose complement is a set of $\mu$-measure zero. This completes the proof of (3.10).

Remark 4. Corollary 1 in Section 2 is a particular case of Theorem 4. In fact, the mapping from $\mathcal{A}:=\mathcal{L}(H)$ to $\mathcal{D}$ which assigns to each operator in $\mathcal{A}$, represented by an infinite matrix with respect to a fixed orthonormal basis $\left(e_{j}: j \geq 1\right)$ in $H$, the "diagonal part" of its representation, is actually a conditional expectation from $\mathcal{A}$ to $\mathcal{D}$. 
REMARK 5. It may happen that there exists no conditional expectation of a von Neumann algebra $\mathcal{A}$ onto its commutative von Neumann subalgebra $\mathcal{B}$. For example, if $\mathcal{A}$ is the von Neumann algebra of all bounded linear operators on $H:=L_{2}(-\infty, \infty)$ and $\mathcal{B}:=L_{\infty}(-\infty, \infty)$ acting on $L_{2}(-\infty, \infty)$ by pointwise multiplication, then there exists no conditional expectation from $\mathcal{A}$ to $\mathcal{B}$ with respect to any faithful, normal state $\phi$. This fact was kindly communicated to us by Professor M. Takesaki in a private letter.

The following theorem is a complement to Theorem 4.

TheOREM 5. Let $H:=L_{2}(0,1)$ equipped with the Borel sets and Lebesgue measure, $\mathcal{A}:=\mathcal{L}(H), \mathcal{B}:=L_{\infty}(0,1)$, and $\left(e_{k}: k \geq 1\right)$ the complex trigonometric system (rearranged into an ordinary sequence). If $\phi$ is defined on $\mathcal{A}$ by (2.1), then there exists a sequence $\left(X_{n}\right)$ in $\mathcal{B}$, bounded in $L_{\infty}$-norm and such that

$$
X_{n} \stackrel{\mathrm{b}, \mathcal{A}}{\longrightarrow} O \quad \text { as } n \rightarrow \infty,
$$

but $\left(X_{n}\right)$ fails to be bundle convergent to $O$ in $\mathcal{B}$.

For example, we may use the trigonometric system $\left\{t \mapsto e^{2 \pi i n t}: n \in \mathbb{Z}\right\}$ as a fixed orthonormal basis in the following rearrangement:

$$
\begin{array}{lll}
e_{1}(t):=1, & e_{2}(t):=e^{2 \pi i t}, & e_{3}(t):=e^{-2 \pi i t}, \\
e_{4}(t):=e^{2 \pi i 2 t}, & e_{5}(t):=e^{-2 \pi i 2 t}, & \ldots
\end{array}
$$

Proof. Since $\mathcal{B}$ acts on $H$ by pointwise multiplication, we have

$$
\left(X_{n} A f\right)(t)=X_{n}(t)(A f)(t) \quad \text { a.e., } n \geq 1, X_{n} \in \mathcal{A}, f \in H .
$$

It follows that

$$
\left\|X_{n} A f\right\|_{2}^{2}=\int_{0}^{1}\left|X_{n}(t)\right|^{2}|(A f)(t)|^{2} d t .
$$

By the reasoning following (2.1), for every $\varepsilon>0$ there exists a natural number $n_{0}=n_{0}(\varepsilon)$ such that

$$
\phi\left(P_{\varepsilon}\right)>1-\varepsilon, \quad \text { where } \quad P_{\varepsilon}:=\sum_{j=1}^{n_{0}} P_{e_{j}} .
$$

Since

$$
P_{\varepsilon} f=\sum_{j=1}^{n_{0}}\left(f \mid e_{j}\right) e_{j}, \quad f \in H,
$$

we have

$$
\left(P_{\varepsilon} f\right)(t)=\sum_{j=1}^{n_{0}}\left(f \mid e_{j}\right) e_{j}(t) \quad \text { a.e. }
$$


Combining (3.15) (with $P_{\varepsilon}$ in place of $A$ ) and (3.16) yields

$$
\left\|X_{n} P_{\varepsilon} f\right\|_{2}^{2}=\int_{0}^{1}\left|X_{n}(t)\right|^{2}\left|\sum_{j=1}^{n_{0}}\left(f \mid e_{j}\right) e_{j}(t)\right|^{2} d t .
$$

By the Cauchy and then the Bessel inequalities, we find that

$$
\begin{aligned}
\left\|X_{n} P_{\varepsilon} f\right\|_{2}^{2} & \leq \int_{0}^{1}\left|X_{n}(t)\right|^{2} \sum_{j=1}^{n_{0}}\left|\left(f \mid e_{j}\right)\right|^{2} \sum_{j=1}^{n_{0}}\left|e_{j}(t)\right|^{2} d t \\
& \leq n_{0}\|f\|_{2}^{2} \int_{0}^{1}\left|X_{n}(t)\right|^{2} d t,
\end{aligned}
$$

that is,

$$
\left\|X_{n} P_{\varepsilon}\right\|_{\infty} \leq \sqrt{n_{0}}\left\|X_{n}\right\|_{2} .
$$

We recall (cf. (2.1)) that

$$
\begin{aligned}
\phi(X) & :=\sum_{j=1}^{\infty} 2^{-j}\left(X e_{j} \mid e_{j}\right)=\sum_{j=1}^{\infty} 2^{-j} \int_{0}^{1} X(t) e_{j}(t) \overline{e_{j}(t)} d t \\
& =\sum_{j=1}^{\infty} 2^{-j} \int_{0}^{1} X(t) d t=\int_{0}^{1} X(t) d t
\end{aligned}
$$

and that bundle convergence in $\mathcal{B}$ coincides with a.e. convergence on the interval $(0,1)$.

Now, it is a routine matter to find a sequence $\left(X_{n}\right)$ of indicators on $(0,1)$ such that

$$
\left\|X_{n}\right\|_{2}=\left\|X_{n}\right\|_{1} \rightarrow 0 \quad \text { as } n \rightarrow \infty
$$

and $\left(X_{n}\right)$ is not convergent to 0 a.e. on $(0,1)$. On the other hand, by $(3.17)$ we have $\left\|X_{n} P_{\varepsilon}\right\|_{\infty} \rightarrow 0$ as $n \rightarrow \infty$, that is,

$$
X_{n} \rightarrow O \text { almost uniformly as } n \rightarrow \infty \text {. }
$$

Since $\left(X_{n}\right)$ is bounded, it follows that $\left(X_{n}\right)$ is bundle convergent to $O$ in $\mathcal{A}$.

REMARK 6. By comparing Theorems 4 and 5 , we see that there cannot exist any conditional expectation with respect to $\phi$ from $\mathcal{A}$ to $\mathcal{B}$, where $\phi$, $\mathcal{A}$, and $\mathcal{B}$ are as in Theorem 5 .

On closing, we raise two problems.

Problem 1. In the conclusion of Theorem 2, is it possible to replace the subsequence $\left(X_{n_{k}}\right)$ by the whole sequence $\left(X_{n}\right)$ ?

Problem 2. In Theorem 4, is it possible to get rid of the condition that the subalgebra $\mathcal{B}$ is commutative and still have conclusion (3.10)? 
Added in proof. The answer to the problem raised in Remark 3 in connection with Theorem 2 is in the negative. In fact, let $H, \mathcal{A}$ and $\mathcal{B}$ be as in Theorem 5 . This time we define $X_{n}(t)$ to be the indicator of the interval $(0,1 / n)$ multiplied by $\sqrt{n}, n=1,2, \ldots$. Analogously to (3.18) in the proof of Theorem 5, we have

$$
\phi\left(\left|X_{n}\right|^{2}\right)=\int_{0}^{1}\left|X_{n}(t)\right|^{2} d t=1, \quad n=1,2, \ldots
$$

So, condition (3.1) is satisfied. Since $X_{n}(t) \rightarrow 0$ a.e. as $n \rightarrow \infty,\left(X_{n}\right)$ is bundle convergent to $O$ in $\mathcal{B}$. On the other hand, no subsequence $\left(X_{n_{k}}\right)$ of $\left(X_{n}\right)$ can be bundle convergent to $O$ in $\mathcal{A}$.

\section{References}

[1] J. Dixmier, Les algèbres d'opérateurs dans l'espace hilbertien (Algèbres de von Neumann), deuxième édition, Gauthier-Villars, Paris, 1969.

[2] E. Hensz, R. Jajte and A. Paszkiewicz, The bundle convergence in von Neumann algebras and their $L_{2}$-spaces, Studia Math. 120 (1996), 23-46.

[3] R. Jajte, Strong Limit Theorems in Noncommutative Probability, Lecture Notes in Math. 1110, Springer, Berlin, 1985.

[4] - Strong Limit Theorems in Noncommutative $L_{2}$-Spaces, Lecture Notes in Math. 1477, Springer, Berlin, 1991.

[5] B. Le Gac and F. Móricz, Beppo Levi and Lebesgue type theorems for bundle convergence in noncommutative $L_{2}$-spaces, in: Oper. Theory Adv. Appl. 127, Birkhäuser, Basel, 2001, 447-464.

[6] A. Paszkiewicz, Convergence in $W^{*}$-algebras, J. Funct. Anal. 69 (1986), 143-154.

[7] M. Takesaki, Theory of Operator Algebras II, Encyclopaedia Math. Sci. 125, Springer, Berlin, 2003.

Barthélemy Le Gac

Université de Provence

Centre de Mathématiques et Informatique

39 rue Joliot-Curie

13453 Marseille Cedex 13, France

E-mail: barthelemy.legac@wanadoo.fr
Ferenc Móricz

Bolyai Institute

University of Szeged

Aradi vértanúk tere 1

6720 Szeged, Hungary

E-mail: moricz@math.u-szeged.hu

Received March 29, 2004;

received in final form September 20, 2004 